\title{
Comparison of Properties of the Collagen Cross-Linked with Aliphatic or Aromatic Reagent
}

\author{
Kazuo Watanabe \\ Nippi Research Institute of Biomatrix, \\ 1-1 Senju-Midoricho, Adachi-ku, Tokyo 120, Japan
}

(Received August 20, 1996)

\begin{abstract}
KEY WORDS Collagen / Cross-Link / Cross-Linkage Structure / Denaturation Temperature / Helix Regeneration
\end{abstract}

Collagen is a very useful protein for constructing artificial organs. ${ }^{1-6}$ When collagen is employed as a biomaterial, chemical cross-links are usually introduced into the collagen molecule to stabilize the collagen triple-helical structure, because a remarkable change in the properties of collagen is caused by the disintegration of the triple-helical structure. ${ }^{7-10}$ In order to prepare a fine cross-linked collagen, the relationship between cross-linkage structure and the properties of the cross-linked collagen was previously examined using six kinds of cross-linking reagents. ${ }^{11}$ Heat-denatured collagens cross-linked with aliphatic reagents regenerated more triple helix than those cross-linked with aromatic reagents by cooling. In the present study, cross-linked collagens were prepared using two kinds of cross-linking reagents that have quite different molecular structures, and several properties of these collagens were extensively investigated.

\section{EXPERIMENTAL}

\section{Materials}

Collagen (solubilized collagen with lime, SCL) was isolated from steer hide using an alkaline solution. ${ }^{12}$ Dimethyl suberimidate dihydrochloride (DMS) was obtained from Wako Pure Chemical Industries, Ltd., and 1 -acetoxypyrene-3,6,8-trisulfonylchloride (APTS) was from Lambda.

\section{Sample Preparation}

The preparation of the collagen cross-linked with DMS (DMS-SCL) was carried out according to the procedure of our previous investigation, ${ }^{11}$ but various amounts of DMS were added to each SCL solution. The amount of the DMS bound to SCL was estimated by quantifying the number of free amino groups in the cross-linked SCL using trinitrobenzenesulfonic acid. ${ }^{13}$ The degree of cross-linking was expressed as the percentage of the closed amino group of SCL by binding the cross-linker. A DMS-SCL solution for spectroscopic measurements was prepared as follows. The lyophilized DMS-SCL was added to $20 \mathrm{mM}$ acetic acid and was continuously stirred at $4^{\circ} \mathrm{C}$ overnight. The DMS-SCL solution was then filtered, and the concentration was adjusted to $0.01 \%$ based on the microbiuret method. ${ }^{14}$

The preparation of the collagen cross-linked with pyrenyl reagent (Py-SCL) was performed as follows.
SCL solution, the concentration of which was about $0.075 \%$, was prepared using $0.1 \mathrm{M}$ phosphate buffer, $\mathrm{pH} 10$. The pyrenyl cross-linking reagent, APTS, was dissolved in acetone, and then various quantities of the acetone solution were continuously and uniformly added to each SCL solution with gentle stirring. The mixture was continuously stirred in the dark at $4{ }^{\circ} \mathrm{C}$ for 17 hours. After the cross-linking reaction had been completed, excess glycine was added to the reaction mixture in order to obstruct the free active groups of APTS by binding glycine. The mixture containing glycine was stirred for additional 3 hours. The reaction mixture was then dialyzed against 10 liters of $1 \mathrm{M}$ acetic acid and five changes of 15 liters of $5 \mathrm{mM}$ acetic acid. The dialysate was lyophilized and stored below $-20^{\circ} \mathrm{C}$. A Py-SCL solution for spectroscopic measurements was prepared as follows. Py-SCL was suspended in $0.01 \mathrm{M}$ sodium hydroxide. The suspension was continuously stirred in the dark at $4^{\circ} \mathrm{C}$ for 10 days and then allowed to stand overnight. The supernatant was quietly transferred on a filter paper to remove some insoluble portion. The concentration of the Py-SCL was adjusted to $0.01 \%$ with $0.01 \mathrm{M}$ sodium hydroxide. ${ }^{14}$ The quantity of the pyrenyl compound contained in the system was determined using a molar extinction coefficient of 1-hydroxypyrene-3,6,8-trisulfonic acid $\left(\varepsilon_{460 \mathrm{~nm}}=2.40 \times 10^{4}\right)$, which has a structure similar to APTS. ${ }^{15}$

\section{Gel Permeation Chromatography (GPC) Experiments}

The procedure for the GPC experiments has been previously described. ${ }^{11}$ Three GPC columns (Shodex OHpak SB-805, Shodex OHpak SB-804, and Shodex Protein KW-804) were employed in the present study to examine the molecular weight distribution of the cross-linked SCL in a high molecular weight region with high resolution. The temperature of the columns was kept at $40^{\circ} \mathrm{C}$ to avoid regeneration of the collagen triple helix.

\section{Circular Dichroism (CD) Measurements}

CD spectra were measured with a JASCO J-600 spectropolarimeter.

The helix-to-coil transition of the cross-linked SCLs, which occurs by increasing the temperature, was observed by monitoring the change in ellipticity. The denaturation temperature of the various cross-linked SCLs was determined according to the method used in our previous 
investigation. ${ }^{11}$

The proportion of the collagen triple helix regenerated from the heat-denatured state by cooling was estimated using the same method as described elsewhere. ${ }^{11,16}$

\section{RESULTS AND DISCUSSION}

We previously prepared six kinds of cross-linked SCLs, the degree of cross-linking of which was sufficiently suppressed, and examined some of the SCL properties. ${ }^{11}$ In order to examine in detail the relationship between cross-linkage structure and the properties of the crosslinked collagen, two types of cross-linking reagents, namely the aliphatic and aromatic reagents, were used with a wide range of cross-linking degree. On the other hand, the distance between the active groups of a crosslinking reagent would also be important to the crosslinking. We have ascertained that the length that can span over one collagen helix pitch $\left(>9.5 \AA^{17}\right)$ is necessary for the cross-linker to efficiently introduce the crosslink into the collagen molecule. ${ }^{18}$ The cross-linking reagents employed in this study react with the amino group of the protein (Scheme 1), and the maximum

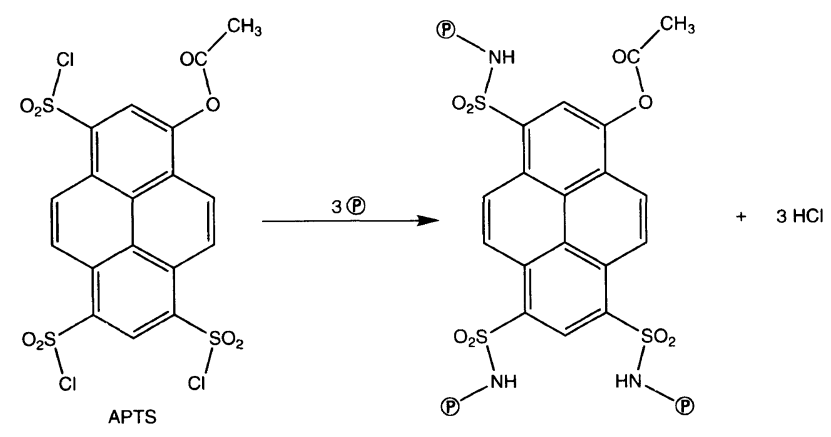

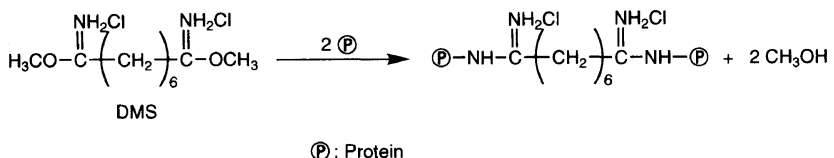

Scheme 1.

lengths between two imino nitrogen atoms interposing each chemical cross-link are presumed to be within the range of 11 to $12 \AA$. Therefore, we selected DMS and APTS in order to examine the structural effects on the properties of the cross-linked SCLs.

The proportion of each chain constituting the collagen helix was examined using GPC. Table I shows the results of the GPC experiments for DMS-SCL, the degree of cross-linking of which was varied. The proportion of the $\alpha$-chain fraction decreased with an increase in the extent of the amino group modification of the SCL side chain. However, a small amount of $\alpha$ chain remained even when the percentage of the amino group modification was above $80 \%$. A large part of the DMS molecule would bind to the SCL side chain with one of the active groups, and another active group was probably free or became inactive. ${ }^{11}$ Some of the high molecular weight fractions above the $\gamma$-chain fraction was found on a chromatogram when the proportion of the amino group modification was above $21.8 \%$. This indicates that intermolecular
Table I. Proportion of each GPC fraction of the SCL cross-linked with DMS

\begin{tabular}{crccc}
\hline $\begin{array}{c}\text { Proportion of } \\
\text { modified } \\
\text { amino group }\end{array}$ & \multicolumn{4}{c}{ Ratio of each GPC fraction $/ \%$} \\
\cline { 2 - 5 } & $\alpha$ Chain & $\beta$ Chain & $\gamma$ Chain & Above $\gamma$ Chain \\
\hline $0^{\mathrm{a}}$ & 67.4 & 25.3 & 7.3 & 0 \\
2.4 & 45.4 & 29.7 & 24.9 & 0 \\
21.8 & 16.9 & 46.2 & 16.5 & 20.4 \\
24.2 & 7.3 & 43.0 & 19.3 & 30.4 \\
33.1 & 6.1 & 39.9 & 20.0 & 34.0 \\
83.9 & 5.5 & 37.7 & 18.0 & 38.9 \\
\hline
\end{tabular}

${ }^{a}$ Unmodified SCL.

cross-linking simultaneously occurred with intramolecular cross-linking within the amino group modification range above $20 \%$. On the other hand, the GPC experiments for Py-SCL could not be performed because very small aggregates were observed using a fluorescence microscope within the solution temperature range above $37^{\circ} \mathrm{C}$. These aggregates were probably produced due to hydrophobic interaction among the pyrene rings.

In order to ascertain whether or not the disintegration of the collagen triple helix occurred by cross-linking, the CD spectra of cross-linked SCLs were analyzed. The collagen triple helix was maintained when DMS was used for cross-linking because the CD spectra of all the DMS-SCLs were the same as the CD spectrum of the uncross-linked SCL (data not shown). In contrast to the CD of DMS-SCL, the CD peak at $221 \mathrm{~nm}$ of PySCL slightly decreased with an increase in the extent of the pyrenyl cross-linking (Figure 1). The helicities of the Py-SCLs, the degrees of amino group modification of which were 22.3 and $82.1 \%$, were 95.8 and $94.0 \%$, respectively. It is likely that the collagen helix partly disintegrated due to the steric hindrance between the pyrenyl cross-linkage and the SCL backbone. Moreover, a slight red shift of the CD peak was observed in the Py-SCL spectrum of which the extent of the closed amino group was $82.1 \%$. This suggests an interaction between the aromatic cross-linkage and the collagen molecule.

The helix-to-coil transition of the cross-linked SCLs occurred due to an increase in temperature. The ellipticity of the cross-linked SCLs markedly decreased within the temperature range of 30 to $40^{\circ} \mathrm{C}$ (data not shown). The denaturation temperature $\left(T_{\mathrm{m}}\right)$ of the cross-linked SCLs was obtained from the denaturation curve ${ }^{11}$ and is shown in Figure 2 as a function of the proportion of the closed amino group in SCL. The $T_{\mathrm{m}}$ of DMS-SCL monotonously increased and was over $40^{\circ} \mathrm{C}$ with an increase in the degree of cross-linking. In contrast to the $T_{\mathrm{m}}$ of DMS-SCL, the $T_{\mathrm{m}}$ of Py-SCL was approximately constant and was lower rather than the $T_{\mathrm{m}}$ of the uncrosslinked SCL. A lowering of the collagen $T_{\mathrm{m}}$ would be caused by the irregularity of the collagen triple helix due to the introduction of the pyrenyl cross-links because the $T_{\mathrm{m}}$ of the triple helix that partly unraveled was about 6-10 degrees lower than the $T_{\mathrm{m}}$ of intact collagen. ${ }^{19}$ On the other hand, the difference in the mode of the chemical bond between each cross-linking reagent and the amino groups of the SCL side chains might be considered as a reason for the difference in the $T_{\mathrm{m}}$ between DMS-SCL and Py-SCL. 


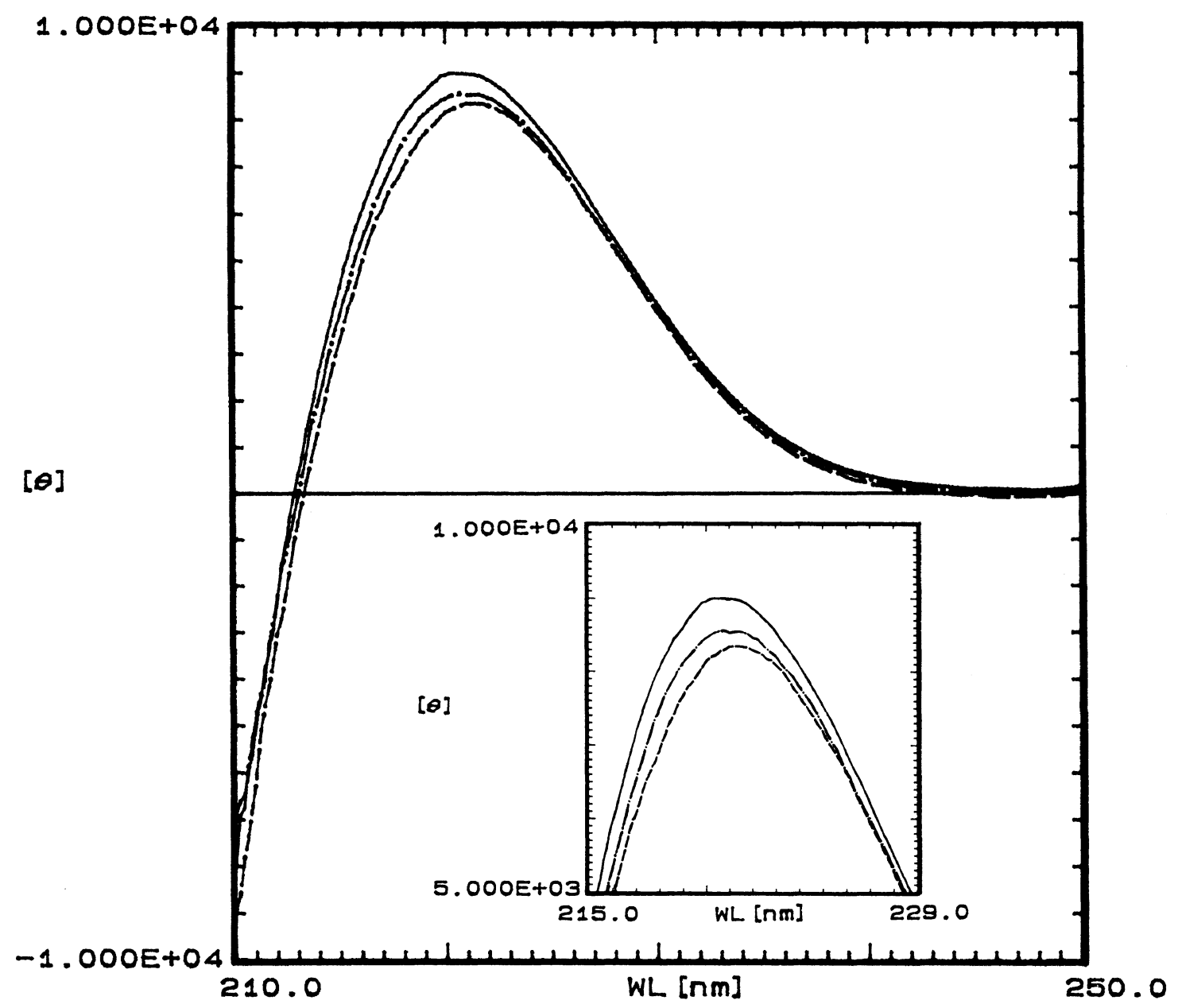

Figure 1. CD spectra of the SCLs cross-linked with APTS. The proportions of the closed amino group are $0 \%(-), 22.3 \%(-\cdot-)$, and $82.1 \%$ $(--)$. The inset is an enlargement of the positive peak centered at $221 \mathrm{~nm}$.

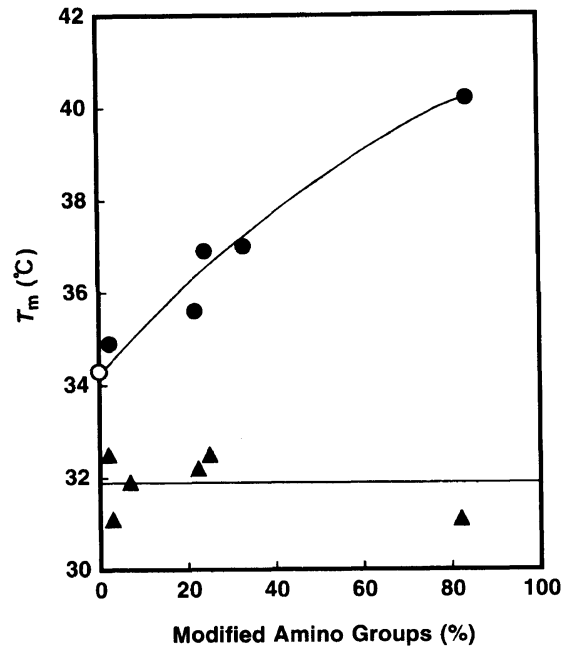

Figure 2. Denaturation temperature of the SCL cross-linked with DMS $(\bullet)$ or APTS $(\boldsymbol{\Delta})$. Open circle is uncross-linked SCL.

The helix regeneration from the heat-denatured state by cooling was examined for DMS-SCL and Py-SCL. The helix content (HC) in the reformed cross-linked SCLs was determined by the ellipticities at $221 \mathrm{~nm} .{ }^{11,16}$ Figure 3 is a graph of the $\mathrm{HC}$ versus the proportion of the closed amino group on the SCL side chain. The HC of both cross-linked SCLs increased with an increase in the degree of cross-linking. In particular, the heat-

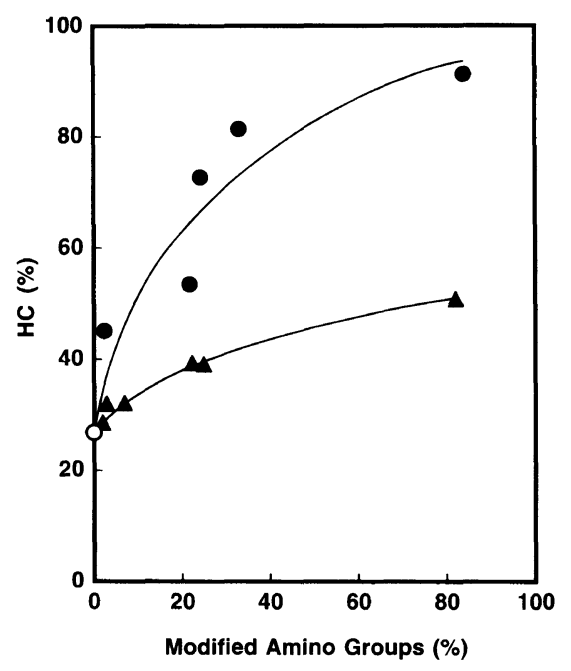

Figure 3. Helix content in the reformed SCL cross-linked with DMS $(\bullet)$ or APTS $(\mathbf{\Delta})$. Open circle is uncross-linked SCL.

denatured DMS-SCL regenerated more triple helix than the heat-denatured Py-SCL when the degree of the amino group modification was the same. Similar results were obtained in our previous investigation. ${ }^{11}$ In general, an aliphatic cross-linkage would more efficiently regenerate the collagen triple helix than an aromatic cross-linkage because a bulky cross-linkage probably inhibits the regeneration of the collagen helix due to steric hindrance. 
In conclusion, cross-linked collagens were prepared using DMS or APTS that has an aliphatic or an aromatic backbone, respectively. The $T_{\mathrm{m}}$ of DMS-SCL increased with an increase in the degree of cross-linking, whereas the $T_{\mathrm{m}}$ of Py-SCL was lower rather than the $T_{\mathrm{m}}$ of the uncross-linked SCL for any degree of cross-linking. The HC in the reformed DMS-SCL was larger than the $\mathrm{HC}$ in the reformed Py-SCL for the same degree of cross-linking. We demonstrated that the aliphatic cross-linkage was more effective for the maintenance and regeneration of the collagen triple-helical structure than the aromatic cross-linkage.

\section{REFERENCES}

1. K. H. Stenzel, M. W. Dunn, A. L. Rubin, and T. Miyata, Science, 164, $1282(1969)$.

2. H. Green, O. Kehinde, and J. Thomas, Proc. Natl. Acad. Sci. U.S.A., 76, 5665 (1979)

3. I. V. Yannas and J. F. Burke, J. Biomed. Mater. Res., 14, 65 (1980).

4. E. Bell, H. P. Ehrlich, D. J. Buttle, and T. Nakatsuji, Science, 211, 1052 (1981).

5. M. Koide, K. Osaki, J. Konishi, K. Oyamada, T. Katakura, A.
Takahashi, and K. Yoshizato, J. Biomed. Mater. Res., 27, 79 (1993).

6. Y. Kuroyanagi, M. Kenmochi, S. Ishihara, A. Takeda, A. Shiraishi, N. Ootake, E. Uchimura, K. Torikai, and N. Shioya, Ann. Plast. Surg., 31, 340 (1993).

7. P. Bruckner and D. J. Prockop, Anal. Biochem., 110, 360 (1981).

8. P. Doherty, E. Robinson, and F. S. Walsh, Exp. Cell Res., 179, 233 (1988)

9. M. L. Lu, R. J. McCarron, and B. S. Jacobson, J. Cell Sci., 101, 873 (1992).

10. A. Berman, G. Morozevich, I. Karmansky, A. Gleiberman, and V. Bychkova, Biochem. Biophys. Res. Commun., 194, 351 (1993).

11. K. Watanabe, J. Nakagawa, T. Ebihara, and Y. Okamoto, Polymer, 37, 1285 (1996).

12. T. Fujii, Hoppe-Seyler's Z. Physiol. Chem., 350, 1257 (1969).

13. W. A. Bubnis and C. M. Ofner, III, Anal. Biochem., 207, 129 (1992).

14. R. F. Itzhaki and D. M. Gill, Anal. Biochem., 9, 401 (1964).

15. O. S. Wolfbeis and E. Koller, Anal. Biochem., 129, 365 (1983).

16. T. Hayashi, S. Curran-Patel, and D. J. Prockop, Biochemistry, 18, 4182 (1979).

17. A. Rich and F. H. C. Crick, J. Mol. Biol., 3, 483 (1961).

18. K. Watanabe, J. Nakagawa, T. Ebihara, and Y. Okamoto, Polymer, in press.

19. A. Rossi, L. V. Zuccarello, G. Zanaboni, E. Monzani, K. M. Dyne, G. Cetta, and R. Tenni, Biochemistry, 35, 6048 (1996). 